\title{
Rethinking the Earth in the Solar System
}

\author{
Gianni Donati \\ AIDIC-Associazione Italiana di Ingegneria Chimica, Milano, Italy \\ Email: gia.donati@tiscali.it
}

How to cite this paper: Donati, G. (2017) Rethinking the Earth in the Solar System. Journal of Applied Mathematics and Physics, 5, 631-638.

https://doi.org/10.4236/jamp.2017.53054

Received: February 17, 2017

Accepted: March 20, 2017

Published: March 23, 2017

Copyright (C) 2017 by author and Scientific Research Publishing Inc. This work is licensed under the Creative Commons Attribution International License (CC BY 4.0).

http://creativecommons.org/licenses/by/4.0/ cC (i) Open Access

\begin{abstract}
The recent discovery that the Earth is retarding each year by a fraction of a second its revolution around the Sun led to investigations and speculations about the cause of such a defect in what was thought to be a perfect clock. The emission of thermal radiation by the Sun cannot justify this discrepancy even if a fraction of unknown dark matter is added to increase the Sun mass loss. The increase of distance of Earth/Moon center of mass from the Sun is estimated of the order of one centimeter per year. However experimental measurements suggest values of the order from 5 to 15 centimeters, hard to be measured for the distances involved. To solve this problem, sophisticated orbital analysis has been proposed, changes in the gravitational constant $\mathrm{G}$ have been suggested and more precise mass/distance measurements in the solar system, asteroids included, have been requested. The present paper shows how the use of an elementary model for the Earth/Moon orbit together with a new theory for the gravitational constant G, coherent with Newton law, can solve this problem. The comprehension of gravity, the ultimate unexplained force of the universe, is the key to solve this and the many remaining question marks in the books of physics.
\end{abstract}

\section{Keywords}

Solar System, Sun and Earth Evolution, Gravity, Neutrinos, Relativity

\section{Introduction}

The Sun and the Earth, we are living on, together with the other planets, their satellites and the various asteroids are the part of the universe we know better. This knowledge however changed in the centuries. We were supposed to stay in the center of the universe, before some courageous people named Copernicus, Bruno and Galileo succeeded in forcing the planets to rotate around the Sun and other geniuses like Kepler and Newton were able to write with mathematics the eternal laws of the Universe.

Now some discrepancies are appearing in these laws with the availability of 
more precise experimental data.

During the last century, the solar system was thought as a perfect machine in perpetual motion: the clock was thought so perfect that we used the rotation period of the Earth to measure our time and the distance $r$ from the sun as the Astronomical Unit (AU) to measure inter galactic distances.

The introduction in 1972 of atomic clocks, available since 1956, allowed the use of International Atomic Time (TAI) and the recognition that the Earth is becoming slower each year by a fraction of second in its revolution around the Sun.

The reason of the slowing down of Earth is not clear but this is going to create problems not only of practical and scientific nature but also of legal one, as explained by G.H. Kaplan in his report [1]

Therefore a Coordinated Universal Time (UTC) has been introduced and is adopted by most nations: in practice UTC is a hybrid time scale that is adjusted each year within one second in June or December, when necessary, by international agreement.

The time delay and the increase of the AU cannot be justified by the radioactive and particulate loss of energy of the Sun.

The Sun has luminosity at least of $3.939 \mathrm{w}$ or $4.382 \times 10^{9} \mathrm{~kg} / \mathrm{s}$ and the particle mass loss, neutrino included, [2] is estimated as $1.374 \times 10^{9} \mathrm{~kg} / \mathrm{s}$ giving a figure of $5.75 \times 10^{9} \mathrm{~kg} / \mathrm{s}$ that might appear very large but is only $9.13 \times 10^{-14}$ the mass of the Sun M per year.

To increase this figure, a contribution of invisible particles called axions is hypothesized [3] that can vary from $0 \%$ to $20 \%$ of luminosity.

Axions are a sort of dark matter and, according to Noedirdingler [4], large values might impose unacceptable changes in the recognized evolution of stellar models.

In addition, to increase the effect, a change in the universal constant $G$ is also hypothesized, given that the product M G may be responsible of the whole phenomena.

In what follows we will refer to Noedirdingler [4] because he provides a lot of information and criticism on available experimental data.

He computes through a simple orbital analysis the increase of the Earth orbital radius $r$ or orbital semi-major axis $a_{o}$ and gets a figure in between 1.37 and $1.57 \mathrm{~m} / \mathrm{cy}$ with no axions and maximum axions respectively; the revolution time delay is however very small and around $5.78 \times 10^{-6} \mathrm{~s}$ while the atomic clocks measure fractions of seconds.

Due to the difficulty in measuring such small figures in a limited time span, an effort has been made to revisit ancient data over long periods and to collect these data in a list of positions of one or more solar system bodies as a function of time, called an ephemeris.

For example Pijeva [5] utilizes data from 1961 to 1995 and obtains a rate of change of AU of $5 \mathrm{~m} / \mathrm{cy}$.

From the analysis of all available radiometric measurements of distances be- 
tween the Earth and the major planets, Kazinsky and Blumberg [6] estimate a change in AU of $15 \pm 4 \mathrm{~m} / \mathrm{cy}$.

Excluding other explanations that, with present knowledge, seem exotic (such as secular decrease of the gravitational constant) there is no satisfactory explanation of the detected secular increase of $\mathrm{AU}$, at least in the frame of the considered uniform models of the Universe.

Therefore a lot of data are collected during space missions and, to expand times, also from ancient Chinese reports [7] to represent in the Solar System Ephemeris the past and future positions of the Sun, the Earth/Moon and the nine planets.

Computer models have been developed to fit the best available observations of the position of planets and largest asteroids [8], with simulations that have been extended from $3000 \mathrm{BC}$ to $3000 \mathrm{AC}$.

However complex models cannot justify the discrepancies due to growing errors in planets positions and poorly defined masses and the revolution delay has no explanation.

In what follows we try to restate the problem using a new view of gravity and a simplified model not to hide real phenomena behind complex mathematics.

\section{Rethinking Gravity}

So while we are discussing the effects of solar mass loss, due to solar radiation and particles emissions, there are still plenty of mysteries that gravity poses.

We are discussing about perturbations of gravity without knowing what gravity is.

Einstein imagined that gravitational attraction between masses results from the warping of space and time by the presence of masses and wrote a general equation for gravity in a similar way the equations for electromagnetism have been written by Maxwell in the previous century.

Somebody made the hypothesis that gravitational field is generated by sub nuclear particles called gravitons or by gravitational waves emitted by matter in a similar way thermal radiation is emitted by the surface of solid bodies.

These tiny sub-nuclear particles are similar to neutrino as far as the equivalent mass or the wave properties are concerned.

If this is the case, what is the flux of gravitons/neutrinos from the Sun to force the planets to move in circles and how much matter loses the Earth to cooperate in the dance?

In previous works [9] [10] [11] we suggested that stable nuclei might emit a neutrino-like particle in order to speed up the electron in their orbits with a mechanism similar to beta decay for unstable isotopes, the difference being that the electron is not expelled.

The analysis of proton-neutron distribution in known nuclei allowed the calculation of neutrino flux from matter that surprisingly is quasi constant per gram of matter and of the order of $F_{o}=6.668 \mathrm{E}+20 \mathrm{n} \cdot \mathrm{g}^{-1} \cdot \mathrm{s}^{-1}$.

We were able to rewrite the Newton law with a neutrino mass 
$\mu=1.55277 \mathrm{E}-36 \mathrm{~g}$ and to compute the Gauss constant

$G=6.668 \mathrm{E}-08 \mathrm{~cm}^{3} \cdot \mathrm{s}^{-2} \cdot \mathrm{g}^{-1}$ in function of $F_{o}, \mu$ and other nuclear parameters (see [8] [9] [10] for details).

$G=F_{o} \mu r_{n}^{2} c / 4 m_{o} \quad\left(r_{n}\right.$ and $m_{o}$ nucleon radius and mass, $c=$ speed of light $)$.

$F_{o}$ is constant, as a mean value for matter, but is slightly smaller for light nuclei and therefore $G$ may slightly change with the composition of matter.

The mass of planets and stars, derived from gravitational effects, may be therefore slightly different from the real one.

The same approximation may be present when computing distances with the aid of Newton law.

The problem is now that the Sun loses, by this mechanism, a mass:

$$
\begin{aligned}
& \mathrm{d} M / \mathrm{d} t=-2.05949 \mathrm{E}+18 \mathrm{~g} \cdot \mathrm{s}^{-1} \text { or } \\
& \mathrm{d} M / \mathrm{d} t / M=-\alpha=-F_{o} \mu-\alpha_{o}=-1.0354 \mathrm{E}-15 \mathrm{~s}^{-1}
\end{aligned}
$$

compared to $-5.75 \mathrm{E}+12 \mathrm{~g} \cdot \mathrm{s}^{-1}$ and $\alpha_{o}=-2.891 \mathrm{E}-21 \mathrm{~s}^{-1}$ computed by surface radiation emission.

On the other hand the Earth loses:

$$
\begin{aligned}
& \mathrm{d} m / \mathrm{d} t=-6.26003 \mathrm{E}+12 \mathrm{~g} \cdot \mathrm{s}^{-1} \text { or } \\
& \mathrm{d} m / \mathrm{d} t / m=-\beta=-F_{o} \mu=-1.0354 \mathrm{E}-15 \mathrm{~s}^{-1}
\end{aligned}
$$

Compared to the null loss by the very small surface radiation emission.

\section{Elementary Orbital Analisis}

Whether the orbit of Earth Moon is a circle or an ellipse the equilibrium is maintained between the centrifugal force and the attractive force:

$$
\omega^{2} r=G \frac{M}{r^{2}}
$$

If the mass of Earth-Moon $\mathrm{m}$ does not change, the angular momentum $L=m l$ is conserved and the specific angular momentum $I$ is also conserved.

$$
l=\omega r^{2}=k
$$

Equation (2) is the third Kepler law.

Now we have $\mathrm{d} M / \mathrm{d} t=-\alpha M$ and $\mathrm{d} m / \mathrm{d} t=-\beta m$ where $\alpha$ is the specific radiation loss of the Sun (neutrino plus radiation loss) and $\beta$ is the specific neutrino loss of Earth.

$$
\begin{gathered}
M=M_{o} \mathrm{e}^{-\alpha t} \\
m=m_{o} \mathrm{e}^{-\beta t}
\end{gathered}
$$

Due to the mass loss of the Earth-Moon the angular momentum at time $t$ is:

$$
L=m l=m_{o} l \mathrm{e}^{-\beta t}
$$

The mass loss is isotropic and therefore one might suppose that the specific angular momentum is not affected and Equation (2) holds.

$$
l=l_{o}
$$

Alternatively we might suppose that, due to mass loss, the angular momentum 
is not conserved and consequently, after completing an orbit in a time $T$, the specific angular momentum is:

$$
l=I_{o} \mathrm{e}^{-\beta T}
$$

The specific angular momentum lost would be:

$$
l-l_{o}=l_{o}\left(1-\mathrm{e}^{-\beta T}\right)
$$

or in general:

$$
l-l_{o}=\eta l_{o}\left(1-\mathrm{e}^{-\beta T}\right)
$$

If $\eta=0$ we have Equation (6) and with $\eta=1$ Equation (7)

Once l is known, Equation (1) and Equation (2) can be re-arranged to give:

$$
\begin{gathered}
r=l^{2} /(M G) \\
\omega=\left(M G / r^{3}\right)^{0.5}
\end{gathered}
$$

The time $T=2 \pi / \omega$, the radius shift $r-r_{o}$ and time loss $T-T_{o}$ can be computed.

\section{Orbital Calculations}

It is important to define an initial point, not necessarily precise but coherent with subsequent calculations.

$\alpha_{o}=2.891 \mathrm{E}-21 \mathrm{~s}^{-1}$ for Sun radiation loss $\alpha=1.0354 \mathrm{E}-15 \mathrm{~s}^{-1}$ neutrino + radiation.

$\beta=0 \mathrm{~s}^{-1}$ for Earth radiation loss $\beta=1.0354 \mathrm{E}-15 \mathrm{~s}^{-1}$ neutrino.

Given a standard initial point:

\begin{tabular}{ccc}
\hline$G$ & $6.6680000 \mathrm{E}-08$ & $\mathrm{~cm}^{3} \cdot \mathrm{s}^{-2} \cdot \mathrm{g}^{-1}$ \\
$M_{o}$ & $1.9891000 \mathrm{E}+33$ & $\mathrm{~g}$ \\
$m_{o}$ & $6.0460770 \mathrm{E}+27$ & $\mathrm{~g}$ \\
year & $3.1567623 \mathrm{E}+07$ & $\mathrm{~s}$ \\
$r_{o}$ & $1.4959787 \mathrm{E}+13$ & $\mathrm{~cm}$ \\
We derive: $\omega_{o}$ & $1.9903891 \mathrm{E}-07$ & $\mathrm{rad} \cdot \mathrm{s}^{-1}$ \\
$\omega_{o} r_{o}^{2}$ & $4.4544 \mathrm{E}+19$ & $\mathrm{~cm} \cdot \mathrm{s}^{-1}$ \\
\hline
\end{tabular}

With only the Sun radiating and $l=k$ we obtain in a year from Equation (10) and Equation (11) the same results given by Noerdingler [4]: $r-r_{o}=1.37 \mathrm{E}+00 \mathrm{~cm} / \mathrm{y} \quad T-T_{o}=5.78 \mathrm{E}-06 \mathrm{~s} / \mathrm{y}$

With the introduction of neutrino emission we might figure out two extreme situations with the Earth global momentum loss at the end of the year $T$ in between $l-l_{o}=0$ and $l-l o=1.45591 \mathrm{E}+12 \mathrm{~cm}^{2} \cdot \mathrm{s}^{-1}$ with the reduction factor $\eta$ between 0 and 1 (Table 1 ).

For $\eta=0.5$ and $l-l_{o}=-7.28 \mathrm{E}+11 \mathrm{~cm}^{2} \cdot \mathrm{s}^{-1}$ we have the same $r-r_{o}=0.0137 \mathrm{~m} / \mathrm{y}$ computed by Noerdingler [4] without neutrino/graviton emission. 
Table 1. Radius shift and time loss in function of $\eta$.

\begin{tabular}{cccc}
\hline$\eta$ & $\left(l-l_{o}\right) * \eta$ & $r-r_{o}(\mathrm{~m} / \mathrm{y})$ & $T-T_{o}(\mathrm{~s})$ \\
\hline 0 & 0 & 4889.5764 & 2.06356 \\
0.1 & $-1.46 \mathrm{E}+11$ & 3911.6639 & 1.75403 \\
0.2 & $-2.91 \mathrm{E}+11$ & 2933.7513 & 1.44450 \\
0.3 & $-4.37 \mathrm{E}+11$ & 1955.8388 & 1.13496 \\
0.4 & $-5.82 \mathrm{E}+11$ & 977.9263 & 0.82543 \\
0.45 & $-6.55 \mathrm{E}+11$ & 488.9700 & 0.67066 \\
0.499 & $-7.26 \mathrm{E}+11$ & 9.7929 & 0.51899 \\
0.5 & $-7.28 \mathrm{E}+11$ & 0.0137 & 0.49900 \\
0.501 & $-7.29 \mathrm{E}+11$ & -9.7654 & 0.51280 \\
0.55 & $-8.01 \mathrm{E}+11$ & -488.9426 & 0.36113 \\
0.6 & $-8.74 \mathrm{E}+11$ & -977.8988 & 0.20636 \\
0.7 & $-1.02 \mathrm{E}+12$ & -1955.8113 & -0.10317 \\
0.8 & $-1.16 \mathrm{E}+12$ & -2933.7238 & -0.41271 \\
0.9 & $-1.31 \mathrm{E}+12$ & -3911.6364 & -0.72224 \\
1 & $-1.46 \mathrm{E}+12$ & -4889.5489 & -1.03177 \\
\hline
\end{tabular}

However for small variations of $\eta$ around $0.5,\left(r-r_{o}\right)$ is changing dramatically.

For a reduction factor of 0.499 we have an increase of $9.7792 \mathrm{~m} / \mathrm{y}$ in the SunEarth distance while with 0.5 we get the same effect computed with radiation alone.

The difference is in the time loss that, for a wide range of $\eta$, remains in a fraction of a second and many times larger than that of Noerdingler.

The yearly increase of distance between Earth and Sun is instead very sensitive to small angular momentum variations and one could expect that additional phenomena or small asymmetries could influence the result.

These phenomena are not considered in the global angular momentum balance with isotropic mass loss.

\section{Relativistic Point of View}

Neutrinos of mass $\mu$ are emitted with the speed of light $c$ by a body like Earth that goes around the Sun with a speed of $v=2.9776 \mathrm{E}+06 \mathrm{~cm} \cdot \mathrm{s}^{-1}$ and a Doppler effect is expected for neutrinos emitted in the direction of motion and for those in the opposite direction.

The neutrino frequency is $f_{o}=\mu c^{2} / h$ were $c$ is the speed of light and $\mathrm{h}$ is the Plank constant.

In a sphere of mass $\mathrm{m}$, in motion with velocity $v=\omega r$, the flux of neutrinos per unit mass in the direction of motion and in the opposite direction can be easily computed as $F_{o} / 4$.

The frequency in the direction of motion $f n+$ and opposite to it $f n-$ are 
blue shifted and red shifted following Doppler effect:

$$
\begin{aligned}
& f n+=f_{o}((1+v / c) /(1-v / c))^{0.5} \\
& f n-=f_{o}((1-v / c) /(1+v / c))^{0.5} \\
& v / c=\omega r / c
\end{aligned}
$$

The specific angular momentum generated to be subtracted after time $T$ to original one is:

$$
l_{o}-l=F_{o} h(f n+-f n-) r T /(4 c)=F_{o} \mu \omega r^{2} T /\left(1-(\omega r / c)^{2}\right)^{0.5}
$$

The specific angular momentum loss is $\left(l_{o}-l\right)=7.27953 \mathrm{E}+11 \mathrm{~cm}^{2} \cdot \mathrm{s}^{-1}$, almost exactly that computed with Equation (9) with a reduction factor $\eta=0.5$

This representation of the angular momentum loss gives a better inside in the nature of gravitation: the same particles emitted by the Earth that cooperate with those emitted by the Sun to define the curvature of the gravitational field, are those that maintain the Earth on a slowly divergent orbit.

This phenomenological description encourages us to find eventual effects of the Moon in the Earth-Moon system: a calculation has been made for the Moon and Earth separately taking into account the relative velocity and distances from their center of gravity.

The effect of this sophistication on $\left(r-r_{o}\right)$ is negligible and of the order of one millimeter accordingly to the approximations made in the calculations.

In addition, for a given value of $G$, the result does not change with the mass of neutrino $\mu$, the loss of angular momentum being correlated and compensated by the loss of mass of the Earth and Sun.

We were unable to find other phenomena to explain possible increases of Earth-Sun distance and agree with Noedirdingler [4] for a revision of experimental data, possibly taking into account the new meaning of the Gauss constant $G$ and the loss of matter of planets and stars.

The delay of the revolution time can however be computed quite accurately and this is the figure we exactly can measure living on Earth.

\section{Conclusions}

To explain the mystery of why the Earth we are living on is changing its speed with time, we recalled a previous study on gravity that explains Newton law in terms of nuclear properties.

In this view matter is emitting gravitons/neutrinos with known flux that causes a loss of mass from the Sun six order of magnitude greater than that due to surface radiation, with the Earth-Moon participating.

In spite of its relevance, this phenomenon is not disruptive and is causing a loss in the angular momentum that is large in absolute values but is a small fraction of the actual one: angular momentum is not conserved and therefore Kepler law holds with some approximation.

A change in the revolution time of a fraction of second is predicted.

Surprisingly the distance from the Sun is not affected and is the same com- 
puted with the sole surface radiation.

This reinforces the criticism on measurements reported in the literature and gives a possible key for the refinement of calculations.

\section{References}

[1] Kaplan, G.H. (2005) Circular No. 179 of United States Naval Observatory.

[2] Bancall, J.N. (1989) Neutrino Astrophysics. Cambridge University Press, 79.

[3] Sikivie, P. (2005) Axions 05 Conference. http://arxiv.org/abs/hep-ph/0509198

[4] Noerdinger, P.D. (2008) Solar Mass Loss, the Astronomical Unit and the Scale of the Solar System. https://arxiv.org/ftp/arxiv/papers/0801/0801.3807.pdf

[5] Pitjeva, E.V. (2005) High Precision Ephemerides of Planets. Solar System Research, 39, 176-186. https://doi.org/10.1007/s11208-005-0033-2

[6] Krazinsky, G.A. and Blumberg, V.A. (2004) Secular Increase of Astronomical Unit from Analysis of the Major Planets Motion and Its Interpretation. Celestial Mechanics and Dynamical Astronomy, 90, 267-288.

https://doi.org/10.1007/s10569-004-0633-Z

[7] Hilton, J.L. and Seidelmann, P.K. (1988) Analysis of Ancient Chinese Records of Occultations between Planets and stars. The Astronomical Journal, 96.

https://doi.org/10.1086/114900

[8] Ito, T. and Tanikawa, K. (2002) Long Term Integration and Stability of Planetary Orbits in Our Solar System. Monthly Notices of the Royal Astronomical Society, 336, 483-500. https://doi.org/10.1046/j.1365-8711.2002.05765.x

[9] Donati, G. (2004) Il legame nucleare e il peso del neutrino. La Chimica e l'Industria, 62-66. http://www.academia.edu/8243167/

[10] Donati, G. (2006) L'universo invisibile (neutrino, quanti e relativita'). ICPN, 92-97. http://www.academia.edu/8243430/

[11] Donati, G. What Gravity Is. https://www.academia.edu/11425803/

Submit or recommend next manuscript to SCIRP and we will provide best service for you:

Accepting pre-submission inquiries through Email, Facebook, LinkedIn, Twitter, etc. A wide selection of journals (inclusive of 9 subjects, more than 200 journals)

Providing 24-hour high-quality service

User-friendly online submission system

Fair and swift peer-review system

Efficient typesetting and proofreading procedure

Display of the result of downloads and visits, as well as the number of cited articles

Maximum dissemination of your research work

Submit your manuscript at: http://papersubmission.scirp.org/

Or contact jamp@scirp.org 